\title{
Art brut oder die Überwindung der Biomacht ${ }^{1}$
}

\author{
Małgorzata Bogaczyk-Vormayr (UAM Poznań, z.Z. Universität Salzburg)
}

Unbegrenzt ist der/ Zusammenhang Sinn und Denken. Edmund Mach $(1994,68)$

\section{Einleitung: Die rohe Kunst}

Im Jahre 1948 publizierte Jean Dubuffet, Künstler und Kunsttheoretiker, in Cahiers de la Pléiade, einer Literatur- und Kunstzeitschrift, die kurz nach dem Kriegsende gegründet wurde, aber mit ihrem Namen an die erste europäische Künstler-Gruppe erinnerte (La Pléiade, gegründet 1549), einen kurzen Text: Notice. Es war jedoch eigentlich ein Manifest in Form einer Zeitungsanzeige. Dubuffet stellte dort eine These auf, die eine Diagnose der modernen Kunst und des Kunstmarktes sowie eine Prognose für neue Wege und Richtungen der Kunst beinhaltete. Des weiteren richtete er sich an die bevorzugten Gesprächspartner für seinen Plan, die Kunstwelt zu ändern: „Daher fordern wir die Ärzte und Leiter psychiatrischer Anstalten auf, uns bei unserer Umfrage zu helfen, indem sie uns Arbeiten von Personen in ihrer Behandlung anzeigen" (Krajewski 2004, 247). Und er hatte dabei ganz offen angekündigt: „Wenn ihnen diese Arbeiten von ihrer Natur in den Rahmen unserer Suche zu passen scheinen, mögen sie uns nach Möglichkeit entweder die Originale oder Photographien dieser Werke übermitteln" (a.a.O.). Der damals von Dubuffet begründete Begriff Art brut (Dubuffet 1991, 82ff) ist heute eine gängige Bezeichnung für eine Kunstrichtung. Diese bezieht sich auf sehr spezifische Phänomene, bildet aber eine sehr breite, inklusive Definition. In seinen Erläuterungen über die „Vorzüge der Art brut gegenüber der kulturellen Kunst" sagt Dubuffet Folgendes:

Die wirkliche Kunst ist immer dort, wo man sie nicht erwartet! Wo niemand an sie denkt noch ihren Namen nennt. Die Kunst

\footnotetext{
${ }^{1}$ Dieser Aufsatz entstand im Rahmen meines Forschungsprojektes „Art brut - Naïve Kunst - Outsider Art. Eine vergleichende Analyse aus der Sicht der Armutsforschung, Resilienzforschung, Kunstphilosophie und Dialogphilosophie“, welches ich seit März 2015 am Zentrum für Ethik und Armutsforschung der Universität Salzburg realisiere. Das Projekt wird durch Mittel des Österreichischen Austauschdienstes finanziert. Ich bedanke mich beim ÖAD und ausdrücklich beim Projektbetreuer, Prof. Otto Neumaier.
} 
verabscheut es, erkannt und mit Namen angesprochen zu werden. Sonst macht sie sich alsbald davon. Die Kunst ist eine Person, die leidenschaftlich an ihrem Inkognito hängt (Dubuffet 1991, 91).

Art brut zeichnet sich nicht durch einen bestimmten künstlerischen Stil oder eine konkrete Technik aus - sie entsteht nicht entlang jener gestalterischen Regeln, die üblicherweise für die Entstehung eines Werkes gelten. Die Charakteristika der Art brut sind erstens das bedingungslose menschliche Bedürfnis, sich künstlerisch auszudrücken, und zweitens die besondere Lebenssituation der Künstler (Krankheit, Behinderung, eine chronisch schlechte soziale Lage, z.B. Obdachlosigkeit). Deswegen wird später der österreichische Psychiater Leo Navratil in seinem Verständnis von "Art brut“ über die „zustandsgebundene Kunst“ sprechen.

Der Begriff „Art brut", also wörtlich: die rohe Kunst, fand Eingang in die Kunsttheorie mithilfe einer Anzeige, einer Kontaktsuche. Oder präziser: Das ist das eigentliche Manifest von Jean Dubuffet, dass man den bestehenden Boden der Kunstwelt verlassen soll-also die Welt der Galerien und Museen, die in den sich nach dem Krieg schnell erholenden europäischen Großstädten bald wiederum herrschen sollten-und jene Welten, welche bis jetzt marginalisiert, isoliert und sogar vernichtet wurden, erforschen und schützen soll. Ist aber überhaupt die Kunst-ein Werk als Ergebnis des künstlerischen Schaffens, eines persönlichen Prozesses-anders als eine rohe, d.h. authentische, als eine vom eigenen, existenziellen Zustand unabhängige zu denken? Ich erwähne hier eine Anekdote: Vor ein paar Wochen arbeitete ich in einem Atelier, Tisch an Tisch, neben Franz Krummholz, Zeichner und Maler aus der Kunstwerkstatt der Lebenshilfe Gmunden/Österreich, und wir unterhielten uns manchmal, immer auf seine Anregung hin, über die Kunst. Plötzlich wendet Franz seinen konzentrierten Blick von seiner Zeichnung ab und spricht mich laut und aufgeregt an: „Hermann Nitsch! Hermann Nitsch! Nur Blut. Tiere und Blut. Ein Schlachthof! Kein Schock, eine Langweile! Ein langweiliger Typ, Hermann Nitsch." Trotz meiner großen Anerkennung für das internationale Renommee von Herman Nitsch kann ich die Ausrufe von Franz Krummholz gut verstehen: der wichtigste Punkt ist die von ihm erwähnte „Langweile“-die penetrante Wiederholung von bereits öffentlich anerkannten Motiven, die durch (Über-)Produktivität erreichte Sättigung bedeutet ein Abhandenkommen der schöpferischen Kraft. Das deckt sich mit dem Kern der Polemik von Jean Dubuffet:

Wir forschen nach künstlerischen Arbeiten wie Gemälden, Zeichnungen, Statuen und Statuetten, die nicht (oder möglichst wenig) die Werke in den Museen, Salons und Galerien imitieren; welche aber umgekehrt den menschlichen Hintergrund und die spontanste und persönlichste Erfindungskraft herausfordern; 
Produktionen, deren Urheber alles (Erfindung und Ausdrucksmittel) aus seinem eigenen Wesen, seinem eigenen Antrieb und seiner eigenen Stimmung zog, ohne Sorgen um die herkömmlichen Möglichkeiten, ohne Rücksicht auf die gebräuchlichen Konventionen. Solche Werke interessieren uns selbst dann, wenn sie summarisch und unbeholfen ausgeführt sind. ... Die gewöhnliche Kunst tritt pompös in den Salons und Galerien auf, sie geht nur vor den Augen der meisten durch und erhält den Namen Kunst, weil sie alles andere ausschließt. Sie erscheint uns von ärmlichem Inhalt, der sich auf ewige Wiederholungen und Imitationen beschränkt und dessen schöpferischer Anteil fast gegen Null geht. Wir halten sie für ein parasitäres Surrogat, das die künstlerische Schöpfung nachäfft, ohne sie zu vollbringen, indem sie die Wörter in zufälliger Ordnung und nach sinnlosen theoretischen Systemen ersetzt (Krajewski 2004, 246).

Im vorliegenden Text möchte ich die Herausforderungen und Hoffnungen, welche Dubuffet formulierte, auf den Prüfstand stellen. Seine Absichten geben nicht nur Einblick in seine eigene künstlerische Position, sondern sie wachsen aus dem Zeitgeist heraus: Zum einen entsprachen sie den Idealen der europäischen Moderne (z.B. die Ereignisse der Weltausstellungen mit dem Salon der Unabhängigen, wo im Jahre 1890 der erste Autodidakt, ein sog. Naiver Künstler, Henri Rousseau, ausstellte), zum anderen kamen sie aus der Schuld und Erschütterung, welche der Nationalsozialismus mit sich brachte. Und somit möchte ich mit einer Skizze der Vorgeschichte zur Art brut beginnen, die - mit Michel Foucault gesprochen - die Macht des Klinischen, d.h. die Prinzipien der Normalität und die Ausgrenzung des Anderen beleuchten soll.

\section{Die Andersheit: Jenseits von Normalität und Pathologie}

Als erstes klassisches Werk von historischer Bedeutung für die spätere Auffassung von Art brut wird gemeinhin die Studie von Ambroise Tardieu Étude médico-légale sur la folie aus dem Jahre 1875 angesehen. Die Aufmerksamkeit, welche die Medizin damals für die bildhauerische Betätigung von schizophrenen Patienten aufbrachte, war nicht geringer als jene, mit welcher professionelle Künstler der Belle Époque, der Avantgarde und letztlich der sog. Entarteten Kunst sich der Problematik geistiger Erkrankung widmeten. Schon nach Cesare Lombrosos Buch Genio e follia (1872) stand eine Zeit lang die Frage im Mittelpunkt, inwieweit das künstlerische Genie zumindest am Rande des Irrsinns steht; eine ganz andere (und theoretisch vermutlich bedeutsamere) Frage betrifft hingegen das Schaffen von geistig wie auch körperlich behinderten Künstlern. Mit den Hauptwerken einiger Psychiater, die zur Kunsttheorie des beginnenden 20. Jahrhunderts wesentlich 
beigetragen haben, ist die Bindung zwischen Medizin und Kunstwissenschaften in der Betrachtung der Außenseiter-Kunst unbestreitbar geworden (wie etwa nach L'art chez les fous von Marcel Réja, 1907; Ein Geisterkranker als Künstler von Walter Morgenthaler, 1910; Bildnerei der Geisteskranken von Hans Prinzhorn, 1922). Das im Laufe des 20. Jahrhunderts steigende Interesse an den Werken der sog. Außenseiter sowie die sich allmählich etablierende Forschung zur sog. Anderen Kunst/Kunst des Inneren/Naiven Kunst führt uns bis heute in tiefe Diskussionen und Auseinandersetzungen mit den Theorien zur Art brut und deren Wandel.

Wie der Begriff „Art brut“ von Dubuffet verstanden und formuliert wurde, umfasste er die nicht-akademische, bildnerische Kunst. Als „rohe Kunst" war damit das spontane Schaffen von künstlerisch nicht ausgebildeten Menschen bezeichnet, von Menschen, die durch ihre soziale Lage (z.B. durch Gefangenschaft, Armut, gesellschaftliche Unangepasstheit) oder durch Krankheit (Psychiatrie-Patienten und Menschen mit geistiger Behinderung) ausgegrenzt waren, sowie die gestalterischen Arbeiten von Kindern. Heute bezeichnet der Art brut-Begriff ausschließlich Arbeiten von Künstlern und Künstlerinnen, die ausgestellt und einem breiten Publikum präsentiert werden - dies ist auch mein eigener kunsttheoretischer Standpunkt. Diesen Kunstbegriff beziehe ich allerdings nicht nur auf Menschen mit geistiger Erkrankung (manisch-depressiv, schizophren usw.), sondern auch ganz allgemein auf geistig und körperlich Beeinträchtigte. Zweitens anerkenne ich als Ausweisung eines Art brut-Künstlers seine chronische Erkrankung, die nicht durch einen künstlerischen Prozess geheilt werden kann - im Einverständnis mit Leo Navratil:

Krankheit ist ein destruierender Prozeß, der aber stets restitutive Vorgänge zur Folge hat. Es sind restitutive Kräfte, die sich in den künstlerischen Werken von Kranken äußern. Diese Kräfte führen zu einer Stabilisierung der Person, aber keineswegs immer zur Heilung: Mitunter fixieren sie die psychotischen Veränderungen und tragen zur Chronifizierung des Leidens bei. Der Wahn der Schizophrenen ist das Ergebnis eines solchen Restitutionsvorganges ... Seit Freud hat man die Vorstellung, dass die Psychose ein primärer Realitätsverlust ist, genauer gesagt: ein teilweiser Verlust jener Bezüge zur realen Umwelt, die für den Menschen von existenzieller Bedeutung sind. Der schizophrene Mensch befindet sich in einem Zustand, in dem er seine subjektive Innenwelt mit der Außenwelt nicht mehr in Einklang bringen kann. Ein wesentlicher Teil dieser Außenwelt ist für ihn irrelevant geworden, ist scheinbar nicht mehr vorhanden (Navratil 1999, 37). 
Ich möchte hier darauf hinweisen, dass man aus heutiger Perspektive genauer aus der wissenschaftlichen Perspektive zur Entwicklung der psychosomatischen Medizin, der Soziologie der Medizin sowie der Kunstgeschichte und Kunstphilosophie - diesem Interesse eine prägende Bedeutung für die Überwindung der absoluten Herrschaft der „Medizin der Arten“ einräumen muss. Dieses erste Hinterfragen der Normalitätsstandards innerhalb der neuzeitlichen Humanwissenschaften verkörperte den Wunsch der Mediziner nach einer fortschrittlichen Entwicklung der Psychiatrie, die tatsächlich als Humanmedizin, d.h. als eine dem Individuum dienende, humanitär wirkende Disziplin anzusehen sei. Ich will jedoch auch darauf hinweisen, dass einige Impulse zu dieser fortschrittlichen Änderung aus der philosophischen Tradition stammten.

In der Tat ist das Thema der außergewöhnlichen Begabung, dabei auch des künstlerischen Schaffens der so bezeichneten Verrückten, der Anderen, der Außenseiter bereits aus den ältesten Zeugnissen der Literatur und der Philosophie bekannt: Dies zeigt sich beispielsweise in der philosophischen Begriffsanalyse zur sog. Alterität. Die Bipolarität des Eigenen und des Fremden hat in den frühesten Zeugnissen der Weltliteratur ihren Platz inne, sie gehört weiters zu den Hauptthemen der ersten Staatskonzepte und politischen Theorien: Der Andere, allos oder xenos, wird zu einem Synonym für barbaros, dem die Gastfreundschaft, der gemeinschaftliche und staatliche Schutz verweigert wird, für andrapolon, für jemanden, der als „Material“ gilt damit waren vorrangig Gefangene oder versklavte Fremde gemeint, die als Arbeitskräfte eingesetzt wurden - und letztlich, allgemein gesprochen, für die Graduierung, die Hierarchiesierung der Menschen nach Herkunft, Rang, Rasse, Alter und Geschlecht. In einer solchen Klassen- und Minderheiten-Bildung werden all diese großen, unterschiedlich marginalisierten Gruppen als ananthropoi-Nichtmenschen/„Untermenschen“ gelten (vgl. Dinzelbacher 1993, $400 \mathrm{ff}) .^{2}$

Der zeitgenössische Theoretiker und Kritiker der Biopolitik, Giorgio Agamben, bemüht sich in seinen zahlreichen Studien, die fortschreitende Politisierung des Lebens darzustellen. Seine große historische, kulturgeschichtliche und sozialpolitische Leistung stellt sich gewissermaßen in die Kontinuität des Werkes von Michel Foucault. Beide Philosophen haben die Mechanismen des totalitären Staates aufgezeigt, nach welchen ihm das unbegrenzte „Recht auf das Leben, auf den Körper, auf die Gesundheit, auf das Glück, auf die Befriedigung der Bedürfnisse" (Foucault 2002, 129) zustünde.

${ }^{2}$ Es ist zu betonen, dass die moderne Alteritätsproblematik (Begegnungsphilosophie, Diskursethik, Friedensforschung, Anerkennungskonzepte u.a.) von den Autoren nicht berücksichtigt wurde. Die kunsthistorische und kunstphilosophische Problematik beschränkt sich dort wiederum auf zwei Sätze, in denen als Vertreter „der Welt des Phantastischen" H. Bosch, P. Brueghel, A. Kubin oder S. Dali erwähnt werden (Dinzelbacher 1993, 444). 
Die Agamben'sche Figur des nackten Lebens werde ich als Terminus in meiner eigenen Reflexion zur Art brut verwenden, in welcher ich auf den historischen Kontext der Klinik eingehen werde. Der Mensch wird in seinen persönlichen Rechten auf das Körperliche reduziert-er ist das Objekt der totalitären Biopolitik, ein Material, welches gewissen Qualitätsstandards, einer allgemeinen Verwertbarkeit Genüge leisten muss. Agamben liefert eine tiefe Analyse zum sog. lebensunwerten Leben, in welcher er aus der 1920 veröffentlichten Schrift des Juristen Karl Binding und des Psychiaters und Neurologen Alfred Hoche (Hoche selbst sah seinen Schwerpunkt in der Medizinethik!) zitiert. Der katastrophale Ruf dieser Arbeit soll uns nicht davon abhalten, uns überhaupt mit ihr auseinanderzusetzen, sondern wir sollten den Mut haben, uns mit den Thesen zur Menschenvernichtung zu konfrontieren. Die Definition des wertlosen Lebens zitiert also Agamben aus dieser Schrift Die Freigabe der Vernichtung lebensunwerten Lebens:

Der Begriff des 'wertlosen' (oder 'lebensunwerten') Lebens kommt vor allem bei Individuen zur Anwendung, die Infolge von Krankheit oder Verletzung als 'unrettbar Verlorene' betrachtet werden müssen und die 'im vollen Verständnis ihrer Lage den dringenden Wunsch nach Erlösung besitzen und ihn in irgendeiner Weise zu erkennen gegeben haben'... Problematischer ist die Lage der zweiten Gruppe; sie 'besteht aus den unheilbar Blödsinnigen-einerlei ob sie so geboren oder etwa wie Paralytiker im letzten Stadium ihres Leidens so geworden sind.' 'Diese Menschen', schreibt Binding, 'haben weder den Willen zu leben, noch zu sterben. So gibt es ihrerseits keine beachtliche Einwilligung in die Tötung, andererseits stößt diese auf keinen Lebenswillen, der gebrochen werden müßte ... (Agamben 2002, 147).

Wir finden hier zwei gut bekannte Motive jeder pseudowissenschaftlichen Klärung und Verteidigung der primitivsten Rassen- und Klassentheorie: Das wohlgemeinte Mitleid mit den Leidenden sowie die elegante, unmerkliche Verschiebung von einem selbstbestimmten, bewussten Subjekt (die Bioethik spricht heute vom „kompetenten Patienten“) zu einem Objekt, welches keinen „Lebenswillen“ habe und dem jedwede Fähigkeit zur Selbstbestimmung abgesprochen wird. Hier haben wir es mit dem nackten, jedes Rechtes und jeden Schutzes beraubten Leben zu tun, welches medizinisch und ethisch, d.h. im Lichte der Wissenschaft und der sozialen Normen, als ein tötbares gewertet wird.

Die erwähnte Schrift-wie Agamben weiter referiert-legte den Grundstein für das „Euthanasie-Programm für den Unheilbaren“, wie der offizielle Titel des im Jahre 1940 von Hitler gestarteten Programms lautete, eigentlich einer Reform zur Hygienisierung, zur Säuberung der Gesellschaft. Es war ein bedeutender Moment für die inneren oppositionellen Strömungen in Nazi-Deutschland: Es war das erste Programm, welches Hitler persönlich von 
Bedeutung schien und gleichzeitig von der Gesellschaft und von der Kirche einstimmig kritisiert wurde, womit es nach 15 Monaten offiziell aufgegeben wurde, was allerdings dennoch viele Morde dieser Art nach sich zog (vgl. Agamben 2002, 150-152).

Einige deutsche und österreichische Art brut-Künstler-Menschen unterschiedlicher Berufe und Beschäftigungen, die aber zu diesem Zeitpunkt Patienten der Nervenkliniken waren und dort zu künstlerischem Ausdruck kamen-befanden sich 1940 auf der Liste des Tötungsprogramms. Was die Sache in ihrer grausamen Ironie noch verdeutlicht, ist die Tatsache, dass einige von ihnen durch den Ersten oder Zweiten Weltkrieg traumatisiert wurden und in der Folge erkrankten. Dieses Schicksal trifft z.B. auf Julius Klingebiel (1904-1965) zu, der in die Wehrmacht aufgenommen wurde und vier Wochen nach Kriegsausbruch an einer akuten Psychose erkrankte. Sofort unterlag er der Zwangssterilisierung, und 1940 wurde er aus der Nervenklinik Langenhagen nahe Hannover in das Verwahrungshaus nach Göttingen verlegt (Spengler et al. 2013, 7).

Das künstlerische Werk von Klingebiel ist seine eigene Zelle im Verwahrungshaus (heute: Asklepios Fachklinikum), welche er 12 Jahre lang (1951-1963) bewohnte und bemalte. Die Phantasmagorie seiner Bilder veränderte die Wände der 9,25 qm großen Zelle zu einem bunten Garten, zum faszinierenden Kaleidoskop mit vielerlei Details: Frauenporträts und -figuren, stilisierte Tiere und Pflanzen, Flusslandschaften und Kleinstädte. In der Klingebiel-Zelle gewinnt man als Beobachter den Eindruck von Souveränität, man betrachtet die Bilder mit ihren unzähligen Details, mit deren Hilfe Klingebiel Tag für Tag, Monat für Monat seine Außenwelt verändert und strukturiert. Man betritt keine Zelle mehr, sondern einen Kunstraum. Klingebiel machte sich diese Zelle, diese fremde und feindliche Welt der Ausgrenzung und Überwachung zu eigen:

In der Geschichte von Julius Klingebiel treffen Menschenverachtung, extreme Unfreiheit und Bedrohung auf Momente der Menschlichkeit und großer künstlerischer Freiheit, in einem entwürdigenden Anstaltsalltag, der heute unvorstellbar ist. Durch seine Kunst blieb Klingebiel Subjekt: Er erschuf sich seine eigene Bildwelt, machte sich 'seine' Zelle zu eigen. So wuchs er über seine eigene Leidensgeschichte hinaus. Große Kunst soll nicht danach bewertet werden, wie gestört der Künstler war oder wie krankhaft seine Erlebniswelt. Gerade in der Klingebiel-Zelle dominiert der Eindruck großer Entwürfe, formaler Gestaltungskraft und künstlerischen Könnens. Wir begegnen hier der gesunden schöpferischen Person (Spengler et al. 2013, 7).

Solch bemalte Räume, die mehr als nur Wohn-, Aufenthalts- oder Genesungsräume sind, die Sicherheit und Zugehörigkeit verkörpern, die ihren 
gewalttätigen, geschlossenen, strafenden Charakter durch die Malerei verlieren, kennen wir von mehreren Art brut-Künstlern. Ein anderes Beispiel dafür ist etwa das Zimmer von August Walla (1936-2001) im Haus der Künstler in Gugging oder der dortige „Garten Wallas“. Gerhard Roth hat zu Recht sein Buch über die Gugginger Künstler Im Irrgarten der Bilder genannt. Wir bewegen uns in Labyrinthen, weil wir hier Gäste sind. Wenn wir aber unsere Aufmerksamkeit steigern, werden uns die dort abgebildete Schönheit und Grausamkeit unserer Außen- und Innenwelt ihre Klarheit, ihre Deutlichkeit aufzeigen. Das Labyrinth wird von unserem menschlichen Wahn und vom Sinn des Menschseins erzählen. Ein solches, von ihm selbst beherrschtes Labyrinth schuf August Walla. Die Auseinandersetzung mit seinen Arbeiten gehört zu den stärksten Erfahrungen, weil er vor unseren Augen das uns gemeinsame Labyrinth erstehen lässt, aus welchem wir nicht flüchten können, mit dem wir uns konfrontieren müssen: Es ist die Welt mit ihren schrecklichsten Nöten - Krieg, Gewalt, Unterdrückung, der schweigende Gott, der uns „nichts schuldig ist“, wie Walla schreibt. ${ }^{3}$

August Walla hat seine psychotischen Anfälle und Halluzinationen schon als Kindergartenkind erlitten. In der Kriegszeit wurde er mit viereinhalb Jahren in die Heilpädagogische Station der Nervenklinik Am Spiegelgrund verbracht, wo er mehr als ein Jahr bleiben musste und wo die Diagnose der psychopathologischen Störung gestellt wurde. Der Leiter der Klinik, Ernst Illing, vermutete eine verschleppte Gehirnentzündung, da er in der Endbegutachtung eine postenzephalitische Wesensänderung diagnostizierte, jedoch das Kind nach Hause schickte. Dr. Illing wurde nach dem Krieg wegen der Durchführung des Euthanasie-Programms verurteilt - mehr als $700^{4}$ Kinder wurden in der Klinik getötet, in Anwendung der billigsten Methoden: Schlafmittel, Unterernährung bis zum Verhungern sowie Unterkühlung (vgl. dazu Navratil 1986, 46; Roth 2012, 70).

Illing wurde für etwa 250 Todesfälle zur Verantwortung gezogen und verurteilt - auch er wurde ermordet. ${ }^{5}$ Wenn wir auf die großen, bunten, von Farben fast strahlenden Bilder Wallas schauen, sind wir augenscheinlich mit Krankheit konfrontiert, welche hinter jeder dieser Zeichnungen steckt: Das kindliche Zeichnen ist mehr eine Karikatur, eine Verkleidung des Schreckens; die lachenden Gesichter sind gleichzeitig große verkrampfte Antlitze, das Lachen hat etwas Bedrohliches. Die Krankheit steckt dahinter - das ist meine ständige Empfindung; ist dieses Gefühl aber nicht eine gewisse Bestätigung

3 Deswegen erscheint mir der von Gerhard Roth stammende Titel sehr treffend: August Wallas Geheim-Schrift-Bild-Zimmer, in: Roth (2012, 69 ff).

4 Es wird auch die Zahl 789 erwähnt; vgl:: U. Weinzier, 14.11.2005 (Zutritt: 22.05.2015).

5 Mit Absicht verwende ich hier diesen Ausdruck, wenn ich über die Hinrichtung auch eines Mörders, eines NS-Verbrechers - spreche. 
dafür, dass wir das Labyrinth, in dem wir so vieles so gut erkennen, möglichst schnell verlassen möchten?

Hier zeigt sich nochmals die schöpferische Präsenz der Andersheit: Ein mithilfe der Kunst beherrschter, gewissermaßen gezähmter Ort ist eine Art von atopos. Der griechische Begriff bezeichnet nicht nur einen Nicht-Ort ( $a$ steht für die Negation; wir haben hier einen Vor-Begriff für „Utopie“), sondern vielmehr jene Ortlosigkeit, welche sich in Nichtzugehörigkeit und Außenseitertum äußert. Das Adjektiv atopos bezeichnet eine Person, die anders, eigenartig, auffällig unkonventionell wirkt, die in gewisser Weise nicht am richtigen Ort ist, oder eine Person, die sich an ihrem Ort-dessen Regeln und Vorschriften-nicht angemessen verhält. Daraus folgt für mich: Diese Person verhält sich nicht gemäß ihrer Zeit, sie ist ihrer Zeit voraus. In diesem Sinne spricht man auch von der philosophischen und künstlerischen Atopie, von der außergewöhnlichen, atopischen Erkenntnis- und Erlebnisqualität. In der Ambivalenz des Atopie-Begriffes spiegelt sich wiederum die Bipolarität des Eigenen und des Fremden: Die Andersheit in ihrer positiven Bewertungdas Andere als das Bereichernde, Neue, Interessante einerseits und das Andere im Sinne von Ablehnung andererseits-als das ungewöhnlich Komische, Merkwürdige, Verunsichernde, als etwas, das uns in Verlegenheit bringt.

Das Ungewöhnliche, das Seltene gilt als abnorm, gilt als etwas, das vom Rande stammt und dort bleiben soll-es sind dies die wohlbekannten und leicht durchschaubaren Ausgrenzungsstrategien. Diese Bipolaritäten, die aus dem Begriff selbst herauszulesen sind, entsprechen einer weiteren Abgrenzung von Normalität und Pathologie. Die Atopie gilt in der Medizin als eine Bezeichnung für eine anormale morphologische Erscheinung oder eine Empfindung (z.B. für die Allergie-man spricht hier von atopischen Reaktionen des Organismus und daraus entstandenen Atopie-Stigmata). Diese gegenseitige Verbindung von Medizinischem und Sozialem, welche dem früheren Naheverhältnis von Biologie und Humanwissenschaften entspricht, hat Michel Foucault ausführlich herausgearbeitet. Es erscheint nützlich, hier eine längere Passage aus seiner Schrift Die Geburt der Klinik zu zitieren:

... das Prestige, das die Wissenschaften vom Leben im 19. Jahrhundert genießen, ihre Modellrolle, sie sie vor allem in den Wissenschaften vom Menschen gespielt haben, hat ursprünglich nichts mit dem umfassenden und übertragbaren Charakter der biologischen Begriffe zu tun, sondern viel mehr mit der Tatsache, daß diese Begriffe in einem Raum verteilt werden, dessen Tiefenstruktur vom Gegensatz zwischen gesund und krank bestimmt war. Wenn man vom Leben der Gruppen und Gesellschaften spricht, vom Leben der Rasse oder selbst vom 'psychologischen Leben', so denkt man nicht in erster Linie an die innere Struktur des organisierten Lebewesens, sondern an die medizinische Bipolarität des Normalen 
und des Pathologischen. Das Bewußtsein lebt, weil es verletzt sein kann, weil es verstümmelt, von seinem Lauf abgelenkt, gelähmt werden kann. Die Gesellschaften leben, denn es gibt unter ihnen kranke, die verkümmern, und andere, die gesund sind und in voller Expansion stehen. ... Zweifellos haben die Wissenschaften vom Menschen Begriffe, die von Biologen gebildet worden waren, übernommen oder zumindest metaphorisch verwendet. Aber ihr Gegenstand-der Mensch mit seinen individuellen und kollektiven Verhaltensweisen und Realisationen-wurde in einem Feld konstituiert, das vom Gegensatz zwischen dem Normalen und dem Pathologischem bestimmt ist (Foucault 1988, 53).

Das bipolare Bild des Gesunden und des Kranken wird als bestehende Grenze angesehen und des Weiteren metaphorisiert-es wird zu einem Mythos anwachsen: Alles muss unter katalogisierte Fälle, Symptome und Krankheitsbilder fallen, alles muss einer Analyse unterzogen werden. Wie aber Foucault öfters betonte, kommt im menschlichen „Willen zur Wahrheit" nicht die Macht des Wortes (z.B. der Analyse), nicht eine Generalisierung und Vor-Strukturierung, sondern die Kraft der Einzelheit zum Ausdruck. Der Mensch ist Träger der Wahrheit und das Wahre kann in einzelnen Aussagen dargestellt werden, aber nicht in einem umfassenden, ordnenden Diskurs abgebildet sein. Foucault macht uns auf eine gewisse Verzauberung, d. $h$. Verfälschung des gesellschaftlichen Lebens aufmerksam: ein Primat des rein Biologischen wird auf das soziale und politische und weiterhin auf das kulturelle Feld übertragen. Die durch beliebige Typologisierungen festgesetzte Trennung von Normalität und Pathologie wird also in das sozialpolitische Feld übertragen, und somit bildet diese Trennung eine ganze Menge von Missbrauchs- und Verletzungsgefahren. In Bezug auf menschliche Schöpfungen, auf Kunst und Kultur, kann es keine auch nur irgendwie relevante Typologie geben.

\section{Das Talent zur Unabhängigkeit: Ein Blick in die Kunstwerkstatt}

Zwei weitere Gugginger Künstler möchte ich hier in Erinnerung bringen, deren Lebens- und Krankengeschichten, deren Werke und künstlerische Betätigung eine für sich sprechende Polemik bilden angesichts der Absicht, eine Trennung zwischen Normalität und Krankheit zu vollziehen. Im Falle von Oswald Tschirtner (1920-2007) sprechen wir ganz deutlich von der künstlerischen Arbeit als Therapie - dies ist nämlich nicht selbstverständlich. Viele Art brut-Künstler brauchen keine äußeren Impulse wie beispielsweise einen Therapeuten, einen Kunsttherapeuten, die Umgebung anderer Patienten, die künstlerisch tätig sind. 
Oswald Tschirtner ist der einzige mir bekannte Art brut-Künstler, dem-wie Leo Navratil wiederholt betonte-jede künstlerische Initiative gefehlt hat: Wenn er zeichnen soll, wartet er auf den Themen-Vorschlag, dann schreibt er den Titel auf das Papierblatt: „2 Menschen in der Sonne“, „2 Menschen geben einander die Hand“, „Zwei Engel“, „Zwei Schwestern“, aber auch: „Ich sitze auf einem Sessel und lese in einem Buch“, „O.T.", oder: „Landschaft”, „Schnee“ u.a. Immer entsteht eine sehr stille Welt, die mit einigen wenigen, strengst reduzierten Tusche-Strichen skizziert wird. „Strengst reduziert“ - es ist dies der Eindruck einer Betrachterin, der sicherlich nicht die konkrete Arbeitstechnik des Zeichners beschreibt. Reduktion meint hier nicht die von Tschirtner selbst ausgewählte oder ihm nahegelegte Technik, sondern die Reduktion ist seine eigentliche Spracheund das bedeutet in keinem Falle eine Einschränkung. Wir betrachten die Zeichnung „Landschaft“: Zwei horizontale Striche mitten durch das Papierblatt und zwei Wörter, die die ganze Ordnung ausmachen-unter dem unteren Strich steht „Erde“, über dem oberen „Wasser“. Aus einem Übermaß ins Wesentliche vorzudringen, ist doch der größte Traum und die größte Leistung der zeitgenössischen Kunst, von der Musik bis zur Bildhauerei. Für die Art brut-Künstler, deren Werke diese reduzierte Sprache des Wesentlichen auszeichnet, ist es keine Bemühung um eine künstlerische Leistung, sondern die Persönlichkeit der Künstler selbst, deren eigene, ungezwungene Ausdrucksform.

Die Menschen- und Engelsfiguren-es sind dies die häufigsten Abbildungen auf Tschirtners Zeichnungen-scheinen sich nur in einem Punkt zu unterscheiden: Die menschliche Figur bleibt offen, die Striche ziehen geradlinig nach unten, meist über die beinahe gesamte Höhe des Papierblattes; ob die Figur mit dem Papierrand (bei Radierungen mit dem Rand der Kupferplatte) endet, ob sie in der Mitte des Blattes situiert ist (seltener) - sie bleibt in ihrer Offenheit unvollendet, wodurch sie zwar eine gewisse Ähnlichkeit aufweist zu einer bewusst nicht fertiggestellten Arbeit, aber eben nicht mit dieser Absicht zu Papier gebracht wurde. Tschirtners Figuren weisen nicht das graphische Element der Geschlossenheit auf, sondern die Linien, die den Rumpf oder-falls vorhanden-die Beine begrenzen, weisen imaginativ weiter nach unten, über den Blattrand hinaus, weiter nach innen vielleicht. Diese einsamen Figuren wachsen wie die Grashalme oder Bäume auf Kinderzeichnungen unvermittelt und wurzellos aus dem Boden. Dem entgegen werden viele Engelsfiguren mit einer gekrümmten Linie am Fußende „abgeschlossen“.

Die Engel scheinen in ihrer Begrenztheit eine Art von Vollkommenheit zu erreichen, sie teilen nicht die Einsamkeit des „bodenlosen“ Menschen. Sehr treffend hat Gerhard Roth auf folgende Motive in Tschirtners Arbeiten hingewiesen: 
Alle Menschen sind komisch. Bei Tschirtners Figuren ist diese komische Seite tragisch. Sie sind sich zwar ihrer Komik bewußt, aber in ihr gefangen. Diese Eigenschaft macht sie GLEICH. Obwohl einsam, sind sie Menschenmassen, denn sie sind dazu verurteilt, zu wiederholen, was andere tun werden. Tschirtner zeichnet die Wiederholung mit (Roth 2012, 109).

Das Komische, also die Andersheit, vielleicht das Unbeholfene, die Verletzlichkeit, die Unsicherheit, wenn die Blicke der anderen auf uns gerichtet sind und wir deren Bewertungen (Analysen, Diagnosen) unterworfen sind. In einem seiner Haupttexte zur Art brut, in der Schrift Anmerkungen für die Schöngeister schrieb Jean Dubuffet über die Verbindung von Kunst und Scherz, dass es zwei Gattungen „vom gleichen Blut" sind: „Das Unerwartete und Ungewöhnliche ist ihre gemeinsame Domäne“ (Dubuffet, 64).

Damit ist genau der Zusammenhang von Komischem und Tragischem gemeint-in der Kunst sowie in der Ironie soll eine gewisse Leichtigkeit spürbar sein, die jedoch die Sackgasse der unschuldigen, belustigenden, belanglosen Witze aufzeigt. Wiederum denke ich hier an die Figuren Wallas an ihr schreckliches Lachen. Der Künstler scherzt (mit uns, mit sich selbst, ein wenig über uns) auf schreckliche Weise-über die Einsamkeit, über die Krankheit, über den Krieg, den Tod und den Teufel-und er zeigt ausdrücklich, dass es bei diesen Themen nichts zum Mitlachen gibt. Dubuffet wird wohl genau solche Scherze gemeint haben: „,.. die ganz starken, die einen auf der Stelle erstarren und zu Stein werden lassen ..." (64-65).

Das Tragische und das Komische der Wiederholung, die Tatsache, dass der Mensch in einer vom Menschen selbst bestimmten Geschichte ein Gefangener ist-auf geniale Weise ist in diesem Blick Tschirtners auf den Menschen die conditio humana diagnostiziert. Nehmen wir als Beispiel die Zeichnung „Heiliger Sebastian“: Wir sehen hier eine menschliche Figur, auf welche unzählige Pfeile gerichtet sind; in Spiegelschrift (es handelt sich um eine Radierung) lesen wir eine Version der Zehn Gebote: : „1. Es ist ein Gott“, „2. Du sollst den Namen Gottes nicht verunehren“, „3. Du sollst Sonn- und Feiertag heiligen“, „4. Du sollst Vater und Mutter ehren, auf daß Du lange lebest und es Dir wohlergehe auf Erden“, „5. Du sollst nicht töten“, „6. Du sollst nicht Unkeuschheit treiben“, „7. Du sollst nicht stehlen“, 8. "Du sollst kein falsches Zeugnis" [weitere zwei Zeilen sind nicht mehr entzifferbar].

Nach meiner Interpretation: Die Pfeile und die neben die männliche Figur geschriebenen Sätze korrespondieren, beides hat eine verletzende Wirkung, die normativen Sätze sind wie die Bogenschützen. Sebastian ist ein verletzter Mensch (ein Mensch par excellence), er leidet hier nicht für seinen Glauben, sondern aufgrund seines "Glaubens", aufgrund der ihn-den Menschen allgemein-betreffenden Vorschriften und Gesetze. Diese sollen vielleicht 
Wegweiser sein, bilden jedoch in Wirklichkeit die Quelle seines Leidens, bedeuten eine Strafe. Die menschliche Welt-so lese ich aus dieser Zeichnung-ist viel eher eine Welt von Gewalt und Schuld, ein Ort der Erschütterung und der Bestrafung, als eine Welt des Mitleids. Und doch präsentiert sich die Welt in den Zeichnungen Oswald Tschirtners nicht auf diese Art-wir hören hier keinen Schrei, wir bleiben vielmehr von der Stille und Ruhe eingenommen. Die vielleicht tatsächlich auf den ersten Blick komischen, naiven, auf jeden Fall harmlosen Figuren in den Zeichnungen Tschirtners verkörpern in der Tat die Ernsthaftigkeit des Tragischen.

Oswald Tschirtner war ein junger Chemie-Student (sein gewünschtes Fach war eigentlich Theologie), als er zur Wehrmacht einberufen wurde. Die schlimmsten Erlebnisse sollte er in Stalingrad sowie im französischen Kriegsgefangenenlager erleiden-und dort, zu Kriegsende, erkrankte er an Schizophrenie. In seiner Krankengeschichte wurde folgende "Diagnose“ eingetragen: „völlig verödet“ (Navratil 1986, 332).

Von 1947 bis zu seinem Tode war Tschirtner durchgehend stationär untergebracht, im Jahre 1954 kam er in die Anstalt Gugging. Der Krieg bleibt ein Thema, welches ihn sein Leben lang begleitet-den Beweis dafür liefern aber nicht Werke, die mit „Krieg“ o.Ä. betitelt wären, sondern seine Zeichnungen und aufgenommene Gespräche zum Thema Ruhe und Frieden. Und was bemerkenswert ist, Tschirtner scheint keinen Impuls zu brauchen, um auf das Thema „Frieden“ zu kommen. Ich zitiere aus einem Gespräch zwischen dem Künstler und seinem Arzt, Navratil:

\author{
$\mathrm{N}$ : Was fehlt Ihnen? Wovon haben Sie zu wenig? \\ T: Ich glaub, ich hab alles. \\ $\mathrm{N}$ : Was wäre Ihr größter Wunsch? \\ T: Frieden soll sein. Frieden. \\ $\mathrm{N}$ : Den haben wir ja. \\ T: Haben wir. Haben wir. \\ N: Der zweitgrößte Wunsch? \\ T: Ruhe soll sein. \\ $\mathrm{N}$ : Haben wir auch. \\ T: Haben wir auch. \\ N: Der dritte Wunsch? (Pause) Ein dritter Wunsch? Hm? \\ T: Frieden. \\ $\mathrm{N}$ : Das war ja der erste! \\ T: War der erste, ja. Wieder Frieden (Navratil 1986, 31-32).
}

Diese zwei Themen-Frieden und Gott-verstehe ich als Wahnmotive des Patienten Tschirtner. Das Interesse an Theologie und die Kriegserfahrungen sind für mich die zwei wichtigsten Orientierungspunkte in seiner Biographie in seiner gesamten Biographie, weil diese ihm bewussten, planmäßigen oder schicksalshaften Komponenten nach seiner Erkrankung die Hauptelemente seiner Wahnideen bilden. Krieg bedeutet eine absolute Vernichtung des 
Friedens-eine Zeit ohne Gott, eine Suche nach dem Gott und ein Erschrecken vor einer gottlosen Welt. Für Tschirtner ist es eigentlich ein Thema: Frieden und Gott als Synonyme. Es waren vor dem Krieg Synonyme für etwas Existierendes, während des Krieges sind es Synonyme für das Abwesende, wonach man sich sehnt, und nach dem Krieg bedeutet dieser eigenartige Doppelbegriff „Gott-Frieden“ etwas Verlorenes. Vielleicht sogar etwas, das enttäuscht hat, das sich zu einem schrecklichen Witz über den Menschen und seinen Glauben gewandelt hat? Die Krankheitsgeschichte Tschirtners ist durch seinen inneren, ruhigen, gar stillen Kampf gekennzeichnet-er kämpft mit der Vorstellung von einer gottlosen Welt oder der Figur eines ungerechten, strafenden, grausamen Gottes. So lesen wir in seinen schlichten Texten: „Friede soll sein. Zum Frieden führe/ mich Jesus. Amen.“ (Navratil 1986, 220); „Die Rose riecht, die Rose sticht. Mein Jesus Barmherzigkeit" (221). Die Ambivalenz des Göttlichen - das Liebesprinzip und die Welt des Nächstenhasses-ist der von Tschirtner erlittene und in seinem Werk ausgedrückte Schmerz. Wir lesen:

\section{SCHMERZ}

Schmerz ist unangenehm. Man spürt ihn kaum. Ich wünsche jedem alles Gute, der bei Schmerz helfen kann. Ich bin für Frieden. Jeder ist für den Frieden. Vielleicht verschwindet so jeder Schmerz. Ich wünsche es. Jedem alles Gute. Tugend nur besteht und bleibt. Mächtiges Herz, Gütiges Herz, bitte für mein armes Herz (Navratil 1999, 32).

Tschirtner fand, wie uns in den Büchern von Navratil und später von Johann Feilacher, dem Nachfolger Navratils in Gugging, vor Augen geführt wird, in Gugging tatsächlich seinen Frieden. Wie Julius Klingebiels Werk dafür ein Beweis ist, dass der Mensch durch seine Genialität der Macht des Klinischen einen Widerstand entgegensetzen kann-bildlich gesprochen: eine Zelle in einen Schutzort verwandeln kann-, so beweist der Aufenthaltsverlauf Oswald Tschirtners in Gugging, wie sich die sog. Klinik zu einem Ort der Inklusion, der Sicherheit und Zuwendung entwickeln kann.

Navratil betonte in Bezug auf Tschirtner, aber auch mit Blick auf den Dichter Ernst Herbeck, dass diese beiden erst arbeiten konnten, wenn sie durch einen äußeren Wunsch und meistens auch durch eine konkrete Themenangabe motiviert wurden. Navratil schreibt, dass es sich bei den Werken von Tschirtner und Herbeck um Externalisierungen handle, welche seine beiden Patienten erst mit therapeutischer Hilfe zustande gebracht hätten (Navratil, a.a.0.), welche also aus langwierigen therapeutischen und künstlerischen Prozessen, innerhalb des ganz normalen Alltags in der Anstalt 
entstanden seien. Gestatten sie uns also auch einen Einblick in die Geschichte von Krankheit und Heilung?

In den zahlreichen Niederschriften zu Tschirtner und Herbeck, welche wir von Navratil haben, wiederholt sich eine Beobachtung, die ich hier besonders interessant finde: Wie bei schizophrenen Patienten sehr häufig, haben auch Tschirtner und Herbeck die Erfahrung eines halluzinatorischen Du. Dieses Du spricht zu ihnen, wird zu einer Stimme im Kopf, verursacht Kopfschmerzen und führt sie letztlich zu entscheidenden Taten. Herbeck kam erstmalig im Alter von 20 Jahren in die psychiatrische Klinik, nachdem er äußerte, dass er von einem Mädchen hypnotisiert wurde 6 , d.h. in seiner Freiheit eingeschränkt, förmlich überfallen wurde. Das war im Jahre 1940, er arbeitete als Hilfsarbeiter in einem Rüstungswerk. Zwei weitere Anfälle ereigneten sich 1942 und 1945-beim letzten Mal unternahm Herbeck einen tätlichen Angriff gegen seinen Vater, der ihn gleichfalls hypnotisieren wolle: „... er drückt mich auf den Kopf ... . Er denkt so scharf und davon bekomme ich Kopfweh" (Navratil 1986, 275).

Tschirtner (dessen dauernde Hospitalisierung 1947 begann, nachdem er mehrere tätliche Angriffe gegen seinen Vater und seine Schwester unternommen hatte) berichtet wiederum in einem Gespräch mit Navratil: „Herr Doktor, ich möchte nur tun, was Gott von mir will. Ich höre eine Stimme, die sagt, ich werde mich umbringen. Aber das ist doch wider das fünfte Gebot!

${ }^{6}$ In seinen Gedichten wird die Schulzeit thematisiert: Als Kind musste er mehrere Operationen einer Lippen-Kiefer-Gaumenspalte ertragen, und er litt unter seinem Aussehen und unter der Sprechstörung. Seine Kindheitserfahrungen waren somit von der Erfahrung der Andersheit und der Ausgrenzung geprägt:

\section{DIE GESPALTENHEIT}

Die Gespaltenheit ist die Arbeit der Ärzte. Diese wird auf den Nenner gebracht die Gespaltenheit ist eine Operation. Und die Kinder wissen es schon.

\section{DIE BÖSEN MÄDCHEN}

Eine Bösartigkeit Sondergleichen. So eine Sauerei. Sie schlagen mich immer Sie bedrohen mich mit dem Messer. Überhaupt Die Mager mag ich nicht mehr. Sie bedrohen mich mit dem Schwert.

\section{DER TOD}

Der Tod ist ganz groß.

Der Tod ist groß

Der Tod ist Grütze.

Der Tod ist schön.

Der Tod ist auch.

Der Tod der Tiere.

Der Tod ist auch dumm.

Ich kann in den Tod gehen.

Der Tod in der Schule als Mädel.

(Herbeck 1992, 61, 119, 11) 
Herr Doktor, ich kenn' mich nicht wieder!“ (Navratil, a.a.O., 214). Die sog. Fernlenkung ist ein immanentes Motiv einer schizophrenen Erfahrung, deswegen fasziniert es, dass wir hier genau von zwei Künstlern sprechen, denen die künstlerische Initiative fehlt. Diese kommt vom Arzt-in Form einer Lenkung: Wir wissen von Navratil, dass Tschirtner und Herbeck nicht durch Psychopharmaka beeinflusst sind-die Medikation wird sogar auf ein Mindestmaß reduziert, um dem Vorwurf der pharmakologisch unterstützen Kunst zu entgehen. Die Berichte, Interviews, auch die Fotos und letztendlich die Werke, die nach einer Interpretation verlangen, sagen aber noch mehr: dass nämlich Tschirtner und Herbeck überhaupt jegliche temperamentvolle Regung und jede Initiative zur Betätigung fehlte (ganz anders als im Falle von August Walla). Warum aber? Wenn dieses Verhalten nicht ausschließlich pharmakologisch bedingt war: war es die Ablehnung dieser fremden, wahnhaften Stimme? Ein Ausweichen vor deren möglichen Verführungen? Die Reduzierung jeder Aktivität, um jede Möglichkeit von Gewalt zu vermeiden? War dieses Gebaren intuitiv oder war es den beiden bewusst und für sie selbstverständlich, wenn auch nicht gesondert artikuliert? Diese unbeantworteten Fragen lassen sich nicht umgehen, mit diesen Fragen beschäftige ich mich, wenn ich die lyrischen Texte beider vor Augen habe. Hier zitiere ich Herbeck:

\section{MEIN HERZ}

mein Herz schlug bis auf den heutigen

Tag wirklich normal. Die Schrift war

kurrent. Der Zaun war hoch die Tigerin war rot.

vor Wut. Die Kaserne lag in meiner Nähe.

Das Herz schlug warm. Das Blut lag

in den Adern

(Herbeck 1992, 115).

Im Falle von Tschirtner und Herbeck haben wir ein ganzes Spektrum von nicht nur medizinisch und kunsttheoretisch, sondern auch sozialethisch relevanten Themen: die Wandlung des Klinischen-vom Ort der Überwachung und Einschränkung zum Ort der Etablierung mithilfe der schöpferischen Tätigkeit, der Einfluss des Arztes auf die künstlerische Arbeit, die vom Arzt gesteuerte Zusammenarbeit seiner Patienten u.Ä. Diese Entscheidung von Navratil, in Gugging ein Haus der Künstler zu gründen, welches keine therapeutische Werkstatt sein sollte, sondern ein Ort für eine von ihm ausgewählte Künstler-Gemeinschaft, kann man unterschiedlich betrachten und bewerten. Üblicherweise wird man in den Diskussionen mit Galeristen und Art brut-Vertretern in Österreich eher eine Kritik an Navratil hören: mehr Exklusion als Inklusion, mehr Einfluss als notwendig, die Monopolisierung der 
österreichischen Art brut, nur die eigene Perspektive und kein Austausch mit anderen Einrichtungen. Gegen die zwei letzten Vorwürfe werde ich hier nicht polemisieren, ihnen jedoch auch nicht Vorschub leisten-beides aus dem gleichen Grund. Wenn wir unsere Analysen nicht auf die zahlreichen Anekdoten aufbauen wollen und die Geschichte des Hauses nicht im Lichte der doch eher persönlich gefärbten Konflikte rund um Navratil sehen wollen, müssen wir feststellen, dass wir über keine Materialien verfügen, die entscheidend darlegen könnten, dass Navratil sein Gugging-Projekt völlig unkritisch geleitet habe, dass er die Polemik von außen und die nicht aus Gugging stammenden Werke ignoriert habe.

Mein Eindruck ist, dass Navratil immer als Psychiater (ich würde sagen: als Psychiater mit dem Schwerpunkt Art brut) gearbeitet hat und somit auf seine eigenen Patienten und deren Werke und Bedeutsamkeit fokussiert war. Zu dem ersten Punkt dieser weithin verbreiteten Kritik an Navratil möchte ich folgende Stellungnahme äußern: Nichts ist für die mir bekannten Art brutKünstler mehr belastend als die künstlerische Einsamkeit, als eine Umgebung, in der keine anderen Künstler tätig sind, wo kein Austausch stattfindet, wo andere Patienten zu einer rein ergotherapeutischen Betätigung angeleitet werden, einer Betätigung also, welche keinen künstlerischen Zweck verfolgt (sondern lediglich das Ziel einer therapeutischen Wirkung vor Augen hat oder die Produktion von Gegenständen) und sich durch keine künstlerische Qualität auszeichnet. Navratil hat also eine Form des Ateliers gegründet, mit allen Barrieren, die damit verbunden sind, auch mit der Einschränkung, dass nicht jeder Patient einer psychiatrischen Klinik imstande ist, Kunst zu produzieren. Wie allgemein in der Kunst, bildet auch innerhalb der Art brut das Talent den ausschlaggebenden Indikator für die Einzigartigkeit und künstlerische Qualität eines Werkes. Wenn ich mich also auf eine Definition beziehen soll, stimme ich mit der Auffassung von „Talent“ bei Johann Feilacher überein:

Talent, das ich als Fähigkeit, einen eigenständigen formalen Weg zu gehen, definieren möchte, ist meiner Ansicht nach eine Fähigkeit, die von psychischer Krankheit unabhängig ist. Natürlich ist es so, dass Menschen in der Psychose anders und für den Laien oft interessant zeichnen, doch bedeutet dieses Anderssein noch keine herausragende künstlerische Qualität. Zeichnungen von Menschen, die an einer Psychose erkrankt sind, unterscheiden sich in der Regel nur wenig voneinander. Psychotiker besitzen im Durchschnitt genausowenig künstlerische Fähigkeiten wie psychisch gesunde Menschen. Nur wenige psychisch kranke Menschen sind also wirklich künstlerisch begabt (Buxbaum 1990, 226).

Das Gugginger Haus der Künstler liefert einen äußerst wichtigen Beitrag in der Etablierung dieses Gedankens, dass an jedem Ort die Bedingungen dafür 
geschaffen werden müssen, in denen der Mensch sich seinem Talent widmen kann—das ist der Moment, in dem man das biopolitische Paradigma der Klinik weit überschreitet. Das biopolitische Paradigma wurde in der Folge langsam und mit Mühe von vielen Institutionen verlassen, was allerdings nicht bedeutet, dass nunmehr die medizinischen Interventionen und die Erfahrungen der einzelnen Patienten von der klinischen Biomacht gänzlich verschont blieben. Ich zitiere ein Gedicht von Herbeck:

\section{DAS GITTERBETT}

Das Gitterbett wird in einer Fabrik gemacht. Es beruhigt die Nerven = der Kranken. Die Herrn Pfleger haben die Verfügung hierzu. Und in einer kurzen Zeit sind die Kranken beruhigt

(Herbeck 1992, 155).

Die Gedichte von Herbeck und die eines weiteren Gugginger Künstlers, Edmund Mach, sind Zeugnisse jener Ambivalenz, welche einem klinischen Ort anhaftet: Erstens: Es sind literarische Texte-die Patienten sind Autoren, sie drücken sich in ihren Texten aus, die Texte werden gelesen, es werden Lesungen organisiert, die Verlage geben die Gedichtbände heraus. Zweitens: Wenn diese Texte das Schreiben und die Klinik zum Thema haben, wird genau diese Ambivalenz in den Mittelpunkt gestellt: „Man soll keine Gedichte schreiben", heißt es in der ersten Zeile in Herbecks Text Zwanzig Ratschläge für Ärzte (a.a.0, 117), die Therapie ist eine Besserwisserei, ist ein Spiel, schreibt Mach, welches erst mithilfe der Elektrizität ernst sein wird, ein Geisteskranker ist „nett u. arm“, „im Geist etwas schlecht, was Professoren anzieht“, ein Gespräch mit dem Arzt wird „zu Visit“, sodass erst der nach Hause entlassene Geisteskranke dort seine Ruhe findet.7

\section{E. Mach: DER GEISTESKRANKE}

Der Geisteskranke ist nett $\mathrm{u}$. arm Spazierend durch die Säale Er redet vor sich hin Und sammelt Worte ohnegleich

Er ist im Geist etwas schlecht was Professoren anzieht die dann helfen und beraten und die Stunde zu Visit machen

Der Geisteskranke später entlassen hat im Heim seine Ruhe 
Viele Gedichte Machs gelten für mich als Texte, in welchen die biopolitischen Standards der Klinik thematisiert werden, in denen deutlich die gleichen Erfahrungen zum Ausdruck kommen, die Foucault der Macht/Ordnung des Diskurses zugeschrieben hat:

\section{DIE THERAPIE}

Ist was leeres was Empfang reines

Die Therapie ist ein Besserwisser

und eine Heilmethode

der Vorgesetzte der die Therapie bringt

ist selbst nicht gescheiter aber das

Wort macht ihn.

$\ldots 8^{8}$

Das Wort macht den Arzt und den Patienten aus - es verhilft zu Abgrenzung, Distanzierung und Rollenverteilung, was man sodann als "Therapie“ bezeichnet. Das Gugginger Haus bildet auch ein historisches Beispiel für eine Lösung, die aus heutiger Perspektive durchaus auch kritisch betrachtet werden muss. Die von lediglich einem Arzt durch Jahrzehnte systematisch betreute und beeinflusste künstlerische Arbeit einiger außergewöhnlich talentierter Art brut-Künstler - das wäre aus heutiger Sicht nicht mehr akzeptabel. Kunstwerkstätten sind nur in jenen Ländern Orte der Inklusion, wo nicht nur die Ressourcen des Gesundheits- und Pflegesystems das erlauben, sondern auch die Etablierung der Art brut im sozialen Bereich (die Idee einer sog. barrierefreien Gesellschaft) wie auch im Kunstbereich

Das Heim ist eingedenkt seiner Ruhe und muntert ihn zum Essen auf.

(Navratil 1999, 143)

E. Mach, 39. Ergänzen kann man mit einem Gedicht von Herbeck:

\section{DER PATIENT}

die Katze ist ein Lamm des Friedens.

So denkt ein Dichter seiner Zeit.

Die im inneren Zeichen eines Psychiaters

einer eigenen Welt gehorcht, - dem Patienten.

Der Arzt zieht die Nummer dann

dem Patienten eine neue Seele an.

der im neuen Geiste einer Krankheit

immer weiterziehen soll.

(Herbeck 1992, 51) 
(Ausstellungen, Verkauf, Sammlungen, Kunsttheorie) es ermöglichen. Diese Werkstätten verdeutlichen den großen Wandel des Klinik-Konzeptes. In diesen Werkstätten trifft man fast ausschließlich auf eine Atmosphäre der Kooperation, der Partnerschaft und der Inklusion. Der Eintritt in ein geschütztes Atelier verläuft selbstverständlich unter gewissen Bedingungen, die sich darauf beziehen, dass das Pflegepersonal bzw. der Arzt oder auch nur der Kunsttherapeut es erlauben müssen. Betrachtet man dies jedoch genau, so ist jedes Atelier ein intimer Ort, und jede Künstlergemeinschaft definiert ihre eigenen Grenzen, die dem Arbeitsprozess und dem Wohlgefühl der dort tätigen Menschen dienen soll. Die Arbeit an einem Kunstwerk ist eine intime Selbsterfahrung und stellt schließlich eine Selbstentblößung dar. Ein Atelier ist par excellence ein Ort der Selbsterfahrung und der Veränderung - kein Schauplatz.

Einige jener kunsttherapeutischen Werkstätten und Ateliers, welche ich regelmäßig besuche und wo ich meine sozialethische These - Inklusion statt Biomacht - überprüfen kann, fungieren als eine Künstlergemeinschaft und werden zum Teil im Sinne von sog. Kollaborationen organisiert. In einigen physischen Werkstätten sind auch vereinsamte Art brut-Künstler tätig - so wird fälschlicherweise der Arbeitsplatz eines einzelnen Künstlers als Kunstwerkstatt wahrgenommen. Ein anderes diskutables Thema ist das Arbeiten nach einer Vorlage: Die Künstler bekommen Bildbände, sie recherchieren im Internet, sie werden von den Kunsttherapeuten dazu aufgefordert, eine ganze Serie zu einem vorgeschlagenen Thema zu gestalten oder gemeinsame Unternehmungen (Spaziergänge, Besuche im Tiergarten, Schwimmbad etc.) graphisch $\mathrm{zu}$ verarbeiten. Derlei therapeutische Arbeitsmethoden können einer künstlerischen Entfaltung völlig zuwiderlaufen oder aber auch keinerlei negative Auswirkung haben. Diese Methoden sind in den rein therapeutisch ausgerichteten Werkstätten selbstverständlich, aber aus dem Blickwinkel der Kunstphilosophie werden sie zu einem sachlichen Problem, wenn man sie nämlich bei Art brutKünstlern anwendet. Auf diese Weise entstehen allerdings trotzdem oftmals selbständige Werke, die in einer großen Entfernung zur Bildvorlage oder zum unterbreiteten Themenvorschlag stehen, wodurch diese folglich nur als Arbeitsanregung fungieren-d.h. hier wirkt in Wirklichkeit das große Talent, die künstlerische Selbständigkeit, kurz gesagt: das Individuum. Man kann aber auch auf belanglose Zeichnungen von Art brut-Künstlern stoßen, die aufgrund der Arbeit nach Vorlage einfachste und häufig nicht besonders gelungene Kopien anfertigen, während ihre ohne Vorlage geschaffenen Werke u.U. eine großartige und berührende künstlerische Qualität aufweisen. Das Thema der teils herausfordernden, teils rückschrittlichen Kopistenarbeit hat Jean Dubuffet wie folgt beleuchtet:

Will man ein Bild kopieren, kollidiert man mit der Schwierigkeit, seine Störungen zu wiederholen. Denn es ist unmöglich, sie geplant 
und vorsätzlich in ihrer Spontanität als Störungen und, was noch dazukommt, an derselben Stelle wie im Original zu reproduzieren. Sie ergeben sich aus tausend Zufällen-der Pinsel hat sich auf seiner Bahn an einem vorstehenden Körnchen festgehakt oder eine Spur Farbe, die nicht trocken war, mitgenommen, wie soll man diese Zufälle wieder ins Werk setzen? Daher kommt es, dass das Kopieren so enttäuschend und überflüssig ist. Ein Bild zu kopieren ist ähnlich wie etwas Handgeschriebenes, eine Unterschrift oder eine Paraphe zu kopieren (Dubuffet 1991, 36).

Faszinierend ist das Thema der Grenze bei jeder Intervention in die Arbeit eines Art brut-Künstlers: was wirkt unterstützend, was überfordernd, was einschränkend? Eine Antwort darauf kann nur individuell gegeben werden, mit Blick auf eine konkrete Künstlerpersönlichkeit, auf die einzelne KünstlerTherapeut-Beziehung, manchmal sogar wird sie sich nur auf eine einzelne Situation beziehen dürfen. Den Modus der Kollaboration könnte man auch mit dem Vorwurf des Paternalismus konfrontieren. Eine solche Kritik könnte ich jedoch nicht unwidersprochen mittragen-sie scheint sich mir sogar aus einer gewissen Vorurteilshaltung abzuleiten. Dass es eine lange und in der Kunstwelt anerkannte Tradition von Kollaborationen gibt-die gemeinsam vorbereiteten Installationen, das einzelne Kunstwerk, welches eine Bearbeitung eines weltbekannten, kanonischen Kunstwerkes darstellt etc.- , soll ein ausreichendes Argument dafür sein, dass Art brut-Künstler untereinander sowie sog. professionelle Künstler mit Art brut-Künstlern kooperieren. Hierbei bestehen zwei Möglichkeiten, die auch dann zum Tragen kommen, wenn nur sog. professionelle Künstler zusammenarbeiten (sic!): Eine gelungene, zwangsfreie, spontane und bereichernde Kooperation oder eine gewisse Lenkung, d.h. eine Steuerung, die zwar vielleicht mit der Absicht einer Hilfestellung angestrebt worden ist, welche aber in Wirklichkeit zum Missbrauch geführt hat.

Vor dieser Frage stehen wir, wenn wir an die von Navratil so offensichtlich gelenkte Zusammenarbeit von Tschirtner und Herbeck denken. Die Zusammenstellung von Tschirtners Zeichnungen und Herbecks Texten würde ich aber in keinem Fall als zu weit gehende Intervention betrachten welche Intentionen der Arzt hatte, bleibt eine nicht rein berufsethische, sondern allgemein moralische Frage, auf die sich keine Antwort finden lässt. Wenn das vorrangige Ziel nicht eine außergewöhnliche Publikation war, sondern die Weiterführung des künstlerischen Arbeitens, wovon der Arzt annahm, dass es eine für seine Patienten förderliche Betätigung sei, so lässt sich daraus kein Recht ableiten, dem Arzt Vorwürfe zu machen. Im Umgang mit diesen Kollaborationen von Herbeck und Tschirtner beschäftigt uns jedoch immer auch eine Frage, die wahrscheinlich unbeantwortet bleiben wird: Wer von den beiden hat auf das schon Gezeichnete bzw. Geschriebene mit einer Zeichnung bzw. einem Gedicht reagiert? Wenn den beiden die 
Initiative zum Arbeiten fehlte, musste der Impuls von Navratil kommen, aber nehmen Herbeck und Tschirtner diesen gleichzeitig auf? Oder war beispielsweise ein Text von Herbeck für Tschirtner mehr Impuls als die Anregungen des Arztes? „Der Traum ist ein klares Licht" liest man bei Tschirtner im Text zu einer Tuschzeichnung, auf der eine Reihe von fragilen, brennenden Kerzen zu sehen sind. Und diese Phrase lesen wir auch in einem Gedicht Herbecks: „Der Traum ist ein Papier/der Traum ist zur Nacht, da kam der Pförtner/der die Tore aufmacht/der Traum ist klares Licht ..." (Herbeck 1992, 8).

Ein interessantes Beispiel einer künstlerisch erfolgreichen und auf menschlicher Ebene sehr berührenden Zusammenarbeit bildet das Werk von zwei Künstlern aus dem Atelier des Diakoniewerkes Gallneukirchen in Oberösterreich: Gerhard Pötscher und Gunter Zehetner. Der erstgenannte ist ein blinder Künstler, welcher kurze Texte in Brailleschrift verfasst, der zweite Künstler mit geistiger Beeinträchtigung ist ein Wörter-Sammler. Es ist eine sehr häufige Tätigkeit von Art brut-Künstlern, den Strom der täglichen Informationen, das Chaos von Daten, Elementen, Gegenständen etc. zu sammeln, dies alles somit vor dem absoluten Verschwinden zu schützen, ihm eine Ordnung aufzuzwingen. Pötscher schreibt in Braille einen Text: „Ein Relief ist das, was man greifen kann. Das ist eine erhabene oder eine aufgeschwollene Geschichte“.

Die Arbeiter des Ateliers produzieren Tafeln in Form eines rechteckigen Reliefs, auf welchen der Satz in überdimensionaler Brailleschrift geschrieben steht. So entstehen Reliefs mit einer „erhabenen Geschichte“ — die sichtbaren und spürbaren Erhebungen der Brailleschrift. Darauf reagiert der Künstler mit folgender Ergänzung: „Irgendwie kann man es mit dem vergleichen, aber nicht ganz." So entsteht am Anfang ein kurzes Gedicht, dann findet eine Werkproduktion statt, welche den Charakter einer Kollaboration/Intervention hat. Die Fixierung des Textes in Form eines Reliefs ermöglicht erst jenen Vergleich, von dem Pötscher spricht: die "aufgeschwollene Geschichte“ als erhabenes, fühlbares Relief. Mit dieser plastischen Form des Textes arbeitet dann der zweite Künstler, den ich an dieser Arbeit einige Tage lang beobachtete-Gunter Zehetner arbeitet ohne jeden Einfluss von außen, er ist im Atelier absolut frei, er wird nicht belehrt, was die Reliefs beinhalten (er wäre auch nicht imstande, diese Information aufzunehmen). Seine Tätigkeit ist von jener Art, über die sich Dubuffet so begeistert äußert-gänzlich

\footnotetext{
${ }^{9}$ Ich zitiere und berichte aus Arbeitsmaterialien, welche mir von Helmut Pum, dem künstlerischen Mitarbeiter im Atelier des Diakoniewerkes Gallneukirchen, freundlicherweise zur Verfügung gestellt wurden. Ich begegnete Gunter Zehetner im Rahmen der Sequenzen 2015, eines jährlichen Workshops „Art brut trifft zeitgenössische Kunst" in Steyrermühl/Österreich. Hiermit bedanke ich mich herzlich bei Helmut Pum und Ferdinand Reisenbichler (Kunstwerksatt der Lebenshilfe Gmunden) für die Möglichkeit der Teilnahme.
} 
unabhängig, intuitiv, spontan, tatsächlich roh, ohne Zielsetzung, aber mit einer Begeisterung für die eigene Leistung, nachdem alle von ihm bearbeiteten Reliefs auf der Wand des Ausstellungsraumes angebracht werden. Was an der Arbeit von Zehetner so faszinierend ist: Er versteht diese Sprache nicht, er weiß nicht einmal, dass es sich um Wörter (wie gesagt: seine eigene Domäne!) handelt, er hat keinerlei Zugang zur Brailleschrift, welchen wir mithilfe einer Übersetzung doch hätten. Er steht in einer doppelten Entfernung zu diesem Text. Und trotzdem nimmt er diese zwei Dimensionen des Reliefs in ihrem wesentlichen Unterschied auf und berücksichtigt diesen auch. Es ist in meiner Betrachtung eine ontologische Stellungnahme von Zehetner, dass er die erhabene Brailleschrift unberührt lässt, sie nicht verändert. Gleichzeitig aber füllt er die weiße Fläche zwischen den Braille-Punkten aus, tagelang mit einem rhythmischen, unveränderten Bewegungsduktus: Mit einem massigen Graphitstift drückt er kräftig Tausende von Punkten in die Polystyrolplatten. Aus diesen Punkten entsteht eine „aufgeschwollene“, gewissermaßen in Bewegung bleibende schwarze Fläche, aus welcher dann die weißen großen Punkte der Brailleschrift herauswachsen.

Wenn man dieses Werk betrachtet, versteht man Navratils Überzeugung vom Kunstwerk als Ergebnis einer Zusammenarbeit und als Externalisierung. Die Mehrdimensionalität eines Werkes kommt zum Vorschein-die Sprache, der Text, das Gedicht, die Fremdsprachigkeit(en), und letztlich die Handbewegungen zweier Künstler, deren Arbeitsverläufe so unterschiedlich, deren Ziele aber durchaus sehr ähnlich sind. Dieses Bedürfnis der Externalisierung und diese Mehrdimensionalität eines Werkes spürte auch Ernst Herbeck:

\author{
EIN TEXT ÜBER EIN GEDICHT \\ Ein Gedicht ist eine \\ Voraussagung. \\ Das Gedicht ist ein \\ Varum. Der Dichter \\ ordnet die Sprache \\ in kurzen Sätzen. \\ Was über ist, ist das \\ Gedicht selber \\ (Herbeck 1992, 196).
}

\title{
4. Schlussworte: Externalisierung ins Zwischen
}

Jede Externalisierung ist meiner Meinung nach eine Form der Individuation.

Hier komme ich $\mathrm{zu}$ einer wichtigen Aussage innerhalb meiner kunstphilosophischen Analyse: Alle Schaffensprozesse sind Methoden der 
Individuation. Den Begriff der Individuation übernehme ich von C. G. Jung, dessen folgende Erläuterungen ich vertrete:

Individuation bedeutet: zum Einzelwesen werden, und, insofern wir unter Individualität unsere innerste, letzte und unvergleichbare Einzigartigkeit verstehen, zum eigenen Selbst werden. Man könnte 'Individuation' darum auch als 'Verselbstung' oder als 'Selbstverwirklichung' übersetzen. ... Da die Psyche keineswegs eine Einheit, sondern eine widerspruchsvolle Vielheit von Komplexen ist, so fällt uns die zur Auseinandersetzung mit der Anima nötige Dissoziation nicht allzuschwer. Die Kunst besteht nur darin, das unsichtbare Gegenüber laut werden zu lassen, ihm gewissermaßen den Ausdrucksmechanismus auf Augenblicke zur Verfügung zu stellen, ohne dabei vom Ekel, den man natürlicherweise vor einem derart absurd erscheinenden Spiel mit sich selbst empfinden mag, oder vom Zweifel an der 'Echtheit' der Stimme des Gegenübers überwältigt zu werden (Jung 1980, 95).

In der Art brut wird diese „Kunst, zu einem Ausdruck zu finden,“ zur engsten Verbindung zwischen dem Ich und dem Werk; die Sprache des Ausdrucks bedeutet eine Vergegenständlichung des Geistigen, die doch keine diagnosegeleitete Klärung sein kann, sondern in der äußeren Form eines Kunstwerkes auf geheimnisvolle Weise verborgen bleibt. Selbstverwirklichung ist nur als Prozess der "Verselbstung“ möglich-dies ist wiederum eine jener Universalien, die sich auf die Individualität stützt. Abhilfe scheint hier die aus der Dialogphilosophie stammende Kategorie des Zwischen zu bringen. Die Sphäre des Zwischen betrachten wir als Begegnungsdimension mit dem Anderen. Das Eigene muss die Grenzen überschreiten-das beobachten wir bei der Begegnung mit Art-brut-Künstlern und Art brutKünstlerinnen auf vielfache Weise: Es gibt Künstler, die das Eigene in das Zwischen einbringen, ohne dass dabei ein innerer Zwang sichtbar wird, und sich dann wieder still aus dem Zwischen zurückziehen (z.B. Oswald Tschirtner). Andere wiederum sind besessen von ihrer Arbeit oder leiden sogar unter der Manie der eigenen Größe, was nicht selten in den Kontext einer psychischen Erkrankung zu stellen ist (wie im Falle von August Walla).10

10 Ein weiteres Beispiel bildet die Person des polnischen Künstlers Nikifor (1895 1968). Die schlimmste Beschimpfung und Strafe, die der obdachlose Künstler an seine Verfolger richtete, die ihn aus den warmen Räumen der Kirche, des Kaffeehauses oder des Bahnhofs vertrieben, lautet: „Nikifor wird nicht mehr malen!“ Der naive Künstler Robert Simon sagt wiederum: „I draw these things because I have to. This art is my lifeline. When I draw I am, for once, at peace with the world. I am relaxed and focused, in a sort of meditative state. Within a few strokes I am one with the pen, paper, and my inner self. I am completely free. I am released from all of the restraints which we as human beings, immersed in our culture, are forced to operate under. Restraints which stifle our freedom and creativity. I can draw whatever my mind can think of. I do not force my mind to draw specific things. I let it guide my drawing as it will. I do not 
Diese „Verselbstung" in der Kunst, die gleichzeitig eine Zwischenwelt eröffnet, ist bei allen Art brut-Künstlern faszinierend und berührend - ob wir uns den Wahn Wallas vor Augen führen oder auch an die Verinnerlichung von Gunter Zehetner denken.

Eine kunsttherapeutische Werkstatt ist eine Sphäre des Zwischen-diese setzt der Biomacht immer einen Widerstand entgegen, weil das Zwischen nur durch Querbeziehungen, nur durch Austausch entsteht: im Gemeinsamen, aber auch durch das Annehmen von Differenzen. Nicht das eine herrschende Diktum ist also der Verständigungsmodus im Zwischen, sondern seine wahre Sprache ist das Anderssein.

\section{Literatur}

Agamben, G. 2002. Homo sacer. Die souveräne Macht und das nackte Leben. Übers. von H. Thüring. Frankfurt am Main: Suhrkamp.

Buxbaum, R. et al. (Hrsg.) 1990. Von einer Wellt zur andern: Kunst von Außenseitern im Dialog. Köln: Du Mont.

Dinzelbacher, P. (Hrsg.) 1993, Europäische Mentalitätsgeschichte. Stuttgart: Kröner Verlag.

Dubuffet, J. 1991. Die Malerei in der Falle. Antikulturelle Positionen (Schriften, Bd. I). Hrsg. von A. Franzke, übers. von E. Kronjäger. Bern - Berlin: Verlag Gachnang \& Springer.

Foucault, M. 1977. Der Wille zum Wissen. Übers. von U. Raulff, W.Seitter. Frankfurt am Main: Suhrkamp.

-——. 1988. Die Geburt der Klinik. Eine Archäologie des ärztlichen Blickes. Übers. von W. Seitter. Frankfurt am Main: Fischer Taschenbuch Verlag.

Herbeck, E. 1992. Im Herbst da reiht der Feenwind. Gesammelte Texte 19601991. Hrsg. von L. Navratil. Salzburg - Wien: Residenz Verlag.

Jung, C. G. 1980. Die Beziehungen zwischen dem Ich und dem Unbewußten. Olten et al.: Walter Verlag.

concern myself with how it will look, indeed I do not have a clue how it will look. I add the colors that attract me at that particular moment. I add color when I feel moved to. Sometimes as I go along, sometimes at various stages throughout the drawing and sometimes near the end. I do not plan myself out of creativity. I am the man in the cave who drew on the wall-because he had to". http://www.outsiderart.co.uk/simon.html. 
Krajewski, M. 2004. Jean Dubuffet. Studien zu seinem Frühwerk und zur Vorgeschichte des Art brut [Dissertation, Friedrich Wilhelm Universität Bonn]. Osnabrück: Der Andere Verlag.

Mach, E. 1994. Triumph des Schockens. Gedichte. Baden b. Wien: Verlag G. Grasl.

Navratil, L. 1986. Schizophrenie und Dichtkunst. München: Deutscher Taschenbuch Verlag.

———. 1999. Art brut und Psychiatrie. Gugging 1946-1986. Wien - München: Verlag Christian Brandstätter.

Roth, G. 2012. Im Irrgarten der Bilder. Die Gugginger Künstler. Hrsg. von D. Bartens und M. Behr. St. Pölten - Salzburg - Wien: Residenz Verlag.

Spengler, A., Koller, M., \& Hesse, D. 2013. Die Klingebiel-Zelle. Leben und künstlerisches Schaffen eines Psychiatriepatienten, Göttingen: Vandenhoeck \& Ruprecht.

Weinzierl, U. 2005. "Ein fruchtbarer Psychiater". Die Welt vom 14.11.2005, http://www.welt.de/print-welt/article177547/Ein-furchtbarerPsychiater.html (Zutritt: 22.05.2015) 


\title{
Małgorzata Bogaczyk-Vormayr (Poznań et Salzburg) \\ Art brut oder die Überwindung der Biomacht \\ (Art brut or the Break-Through on Biopower)
}

\begin{abstract}
In this comprehensive paper, I present the thesis that the clinical biopower-which means biopouvoir according to Michel Foucault-can be countered with the help of artistic ability. In this sense, the psychiatric clinic may turn into a space of inclusion, respect, and true self-unfolding. Following Jean Dubuffet, I give a definition of art brut and analyze some works of German and Austrian outsiders of the $20^{\text {th }}$ century, who succeeded in overcoming life-crises, such as childhood poverty, experiences of war, psychical illness, or social ostracism. I match the biopolitical character of psychiatry clinics against the most recent ideas of art therapy. The remainder of the article is organized in four parts: (1) Introduction: Raw Art, (2) Alterity: Beyond of Normality and Pathology, (3) Talent for Sovereignty, (4) Conclusion: The Between.
\end{abstract}

Keywords: Art brut, outsider art, philosophy of art, biopolitics, art therapy, alterity, inclusion, clinics, illness, trauma, creativity, self-unfolding

Doi: $10.14746 /$ eip.2014.2.7 